\title{
Hepatitis of Halothane: A Fatality to Avoid; A Case Report
}

\author{
Jbili ${ }^{1 *}$, Bibiche $\mathrm{L}^{1}$, Jebbar $\mathrm{N}^{1}$, Maaroufi $\mathrm{A}^{1}$, Diai $\mathrm{A}^{2}$, Chkoura $\mathrm{K}^{2}$, Kechna $\mathrm{H}^{2}$ and Laoutid J ${ }^{2}$ \\ ${ }^{1}$ Pole of anesthesia, resuscitation and emergencies, Meknes Military Hospital, Morocco \\ ${ }^{2}$ Faculty of Medicine and Pharmacy of Rabat, Mohammed V University, Morocco
}

*Corresponding author: Nabil Jbili, Pole of Anesthesia, Resuscitation and Emergencies, Moulay Ismail-Meknes Military Hospital, Morocco

\begin{abstract}
Summary
We report the observation of a 56-year-old female patient, with no specific medical history, operated on for acute cholecystitis. One week after the intervention, she presented a fatal fulminant hepatitis in less than two weeks, associating a deterioration of the general state with jaundice cutaneous mucosal frank, cytolysis and cholestasis important in addition to a deep hypo albuminemia and a very low prothrombin level. The toxicity of halothane has been incriminated due to the negativity of the etiological balance. Like the literature and this clinical case, the still frequent use of halothane in several countries must be reviewed.
\end{abstract}

\section{Introduction}

Halothane is a halogen used for inhalation induction especially in children and the maintenance of anesthesia in adults and children. Its use is less and less widespread given its hepatic toxicity and its unfavorable hemodynamic repercussions, and the advent of new generations of more harmless halogens, however it remains in certain countries. Therefore, it is necessary to rationalize its use and take the necessary precautions.

\section{Observation}

We report the case of a 56-year-old patient, with no specific medical history, who was operated on for acute cholecystitis, the simple post-operative consequences of which allowed discharge in 48 hours. The patient had no specific defects, including no liver disease or atopy. She was not taking any medication and was classified ASA I with a normal preoperative liver and kidney workup. Cholecystectomy was performed by laparotomy under cost, under general anesthesia. A standard induction by a morphine 'Fentanyl ${ }^{\circ}$, a barbiturate 'Thiopental ' and a non-depolarizing curare "Esmeron" at doses appropriate to the patient's weight. The maintenance was carried out with halothane with an equimolar 02/NO mixture. From induction to waking, the patient remained hemodynamically and respiratory stable. The extubation is performed on a table and after an Aldrete score of 10 in the post interventional monitoring room, the patient is transferred to the service. The consequences were simple with resumption of transit the day after the intervention and the exit was decided in 48 hours. One week after the day of the intervention, the patient accused anorexia and asthenia in addition to the appearance of a frank cutaneous mucosal jaundice with dark urine evolving in a context of a pyrexia but especially of a deterioration of the "general condition which motivated the patient to consult urgently. Upon admission, the patient was non-pyretic, time and space oriented and hemodynamically and respiratory stable. His abdomen was un distended and flexible on palpation, no hepatomegaly or splenomegaly. The bowel sounds were audible, and the digital rectal examination was without abnormalities.

The mucosal skin examination revealed frank jaundice and icteric conjunctiva. Cardiovascular, respiratory and neurological examination was without abnormalities. The initial assessment showed a hemoglobin level at $13.7 \mathrm{~g} / \mathrm{dl}$, a hyperleukocytosis at $17940 / \mu \mathrm{l}$ (PNN and monocyte), thrombocytopenia at $89,000 / \mu \mathrm{l}$, a low prothrombin (TP) rate at $34 \%$ and a time active cephalin (TCA) at $36 \mathrm{sec}$, cytolysis with ASATs (SGOT)=760 IU/L (21 times normal value) $\mathrm{ALAT}=1042 \mathrm{IU} / \mathrm{L}$ (25 times normal value), cholestasis with total Bilirubin at $246.17 \mathrm{mg} / \mathrm{l}$ (20 times the normal value), direct bilirubin=173.6mg/l (34 times the normal value) gGT=293 U/L, $\mathrm{PAL}=199 \mathrm{U} / \mathrm{L}$ (1.2 times the normal value); the blood sugar level was correct and the normal lipasemia at 46ui/l. On the infectious level, apart from a CRP of $145.09 \mathrm{mg} / \mathrm{l}$, the ECBU remained sterile, 
the chest X-ray did not show any suspected parenchymal focus and the blood cultures remained sterile. In addition, hepatic serologies were found to be negative. The abdominal ultrasound showed a simple intraperitoneal effusion blade without dilation of the intra and extra hepatic bile ducts and the Bili-MRI was normal.

The therapeutic management consisted of rehydration with eviction of all hepatotoxic drugs and monitoring of the count of the blood formula and particularly the platelets, of the hydro electrolytic assessment and the blood case. Evolution was marked by the accentuation of the mucocutaneous jaundice and concentrated urine and the worsening of the general state with a deterioration of the consciousness. On the biological level, there has been a gradual drop in the prothrombin level from $34 \%$ (on admission) to $16 \%$ on the tenth day after admission, deep hypo albuminemia at $20 \mathrm{~g} / \mathrm{l}$, with worsening of cytolysis, cholestasis and thrombocytopenia. On the contrary, hemoglobin and blood sugar remained stable and kidney function was preserved.

\section{Discussion}

Since its introduction on the market, halothane has been implicated in the occurrence of postoperative hepatitis. Its hepatotoxicity has been discussed for a long time, but currently, there are many arguments that confirm its existence in humans and animals. This diagnosis is more evocative in the absence of other arguments in favor of another etiology $[1,2]$. These arguments are based on retrospective and prospective Studies: -retrospective studies concerning the circumstances of the occurrence of postoperative hepatitis; the oldest and most important of which is the National Halothane Study, covering 856,500 anesthesia's performed in the USA from 1959 to 1962. This study showed the existence of unexplained massive hepatic necrosis after administration of halothane and that the frequency of these hepatitis was low, seven for 254,896 halothane administrations. Other studies have confirmed that 82 to 95 percent of these hepatitis infections are seen primarily after multiple administrations. 100 of cases and spaced less than 28 days in 55 to 75 p. 100 of hepatitis observed after multiple exposures. - and prospective studies; measuring biological parameters after halothane administration $[1]$.

The occurrence of post-halothane anesthetic hepatitis is often spaced less than 28 days after multiple exposures in $65 \%$ of cases [1], but in some cases, the occurrence may be early, within days or even hours. follow, especially in the event of repeated and closeup exposures [2-4]. After exposure to a toxic agent, hepatoxicity is generally dose-dependent and therefore predictable, appearing after a short delay (1-12 weeks). More rarely, it is idiosyncratic, dose-independent and appears with a longer latency period (up to 12 months) [5]. The hepatic toxicity of halogenated agents of the Immunoallergenic type is linked to the production of trifluoroacetic acid [6,7], These terminal derivatives of metabolism form with hepatic cytosolic proteins a neoantigen for which the organism produces immunoglobulins G specifically directed against hepatocytes [6]. The greater the metabolism of the halogenated agent, the greater the quantity of neoantigens formed and the higher the risk of cytolytic hepatitis. Thus, when using halothane which is metabolized to $20 \%$, its incidence is around $1 / 10000$ anesthesia in adults. It is believed to be lower in children, around $1 / 200,000$, although the metabolism of halothane does not differ between children and adults [6].

The discovery of a hypersensitivity mechanism during halothane hepatitis constitutes an interesting diagnostic contribution, as highlighted in the general review of GOLDFARB $[5,2]$. Other authors have noted the presence of antibodies to halothane-treated rabbit hepatocytes in the serum of patients with severe halothane hepatitis. Unfortunately, these explorations are not common practice and do not represent a formal diagnostic criterion [4,2]. In addition to individual vulnerability [5], Several contributing factors have been implicated in the occurrence of halothane hepatitis, hypovolemia, hypoxia, obesity, female sex and, above all, repeated exposure to this anesthetic. However, according to GOEDFARD, at least $5 \%$ of patients have an increase in SGPT after the first administration of halothane [4,2]. Preexisting liver damage, ischemic liver following an episode of low peripheral flow and the type of surgery, those which cause manipulation of the hepatic compartment or of long duration are also incriminated $[3,5,8]$. The use of a barbiturate in anesthetic induction, as an enzyme inducer, also promotes the appearance of hepatitis [2].

Chemotherapy promotes the severity of halothane hepatitis and disrupts the metabolism of antimitotic by potentiating their toxic effect. In 1980, SPIEGEL et al. reported two reports of fulminant hepatitis following heavy chemotherapy and halothane anesthesia $[2,9]$. The risk of halothane hepatitis is low in children (below the age of 20) [4] and after the first anesthesia [9]. In adults this risk is estimated at fulminant hepatitis for 35,000 halothane anesthesia's. For most of these toxic drugs or not, the management, after elimination of the toxic is symptomatic [5]. The peculiarity of the observation that we report is the absence of favorable factors apart from the female sex and the use of a barbiturate type thiopental at induction. The intervention was carried out without any notion of hypovolemia or hemodynamic or respiratory instability and the research in the history did not find any previous exposure to halogens. In addition, biological and radiological explorations have not objectified any etiology which could explain this hepatic insufficiency suggesting the exposure to halothane. Indeed, given the gravity of this attack, its rapidity of development and the absence of predictive tests, it would be desirable, in adults as in children, to space out the administration of halogens over time or use other halogens that would cause little immunoallergic hepatitis $[3,9]$. And if there is no restriction on the repeated use of halogens in children, this judgment must be reviewed [4]. In addition, like Spiegel et al, it is preferable to strictly ban the use of halothane in patients who have received or are to receive antimitotic treatment $[9,10]$.

- I declare that the text has been read and approved by all the authors.

- All authors declare that they have no conflict of interest. 


\section{References}

1. Bunker JP, Forrest WH, Mosteller F, Vandam LD (1969) National Halothane Study. A study of the possible association between halothane anesthesia and postoperative necrosis. Washington DC Government Printing Office US.

2. Goldfarb G (1982) Hépatite à L'halothane. Ann Fr Anesth Réanim 1(6): 629-633.

3. Formes P, Lecomte D (1991) Hépatite A I'halothane: risque lié à la drépanocytose SC. Ann Fr Anesth Réanim 10: 221.

4. Mjahed K, Sadraoui A, Salmi S, Laraki M, Benaguida M (1997) Hépatite fatale chez un jeune enfant: rôle probable de l'halothane. Ann Fr Anesth Réanim 16(5): 5331-5333.

5. Mégarbane B, Deye N, Baud F (2007) Toxic hepatitis: Mechanisms of toxicity and specific pharmacological agents. Société de réanimation de langue française 16: 632-642.
6. Odin I, Nathan N (2005) Anesthésiques halogénés. EMC-Anesthésie Réanimation 2: 79-113.

7. Treille C, Laturaze T, Aubert H, Rachaii M (1982) Les h patopathies médicamenteuses en milieu hospitalier: Etude épidémiologique et étiologique rétrospective. A propos de 29 case. La Revue de Médecine interne 3(1): 101-109.

8. Eghtesadi AP, Sohrabpour A, Vahedi H, Saberi FM (2008) Halothane hepatitis in Iran: A review of 59 cases. World J Gastroenterol 14(34): 5322-5326.

9. Gounot R, Perret PH, Metafiot H, Pouyau G, Freycon F, et al. ((1984) Hépatite postanesthesique: Responsabilité de I 'associationhalothaneantimitotiques. Ann Fr Anesth Reanim 3(3): 212-215.

10. Peiris LJ, Agrawal A, Morris JE, Basnyat PS (2012) Isoflurane hepatitisinduced liver failure: a case report. Journal of Clinical Anesthesia 24(6): 477-479.

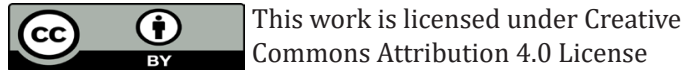

To Submit Your Article Click Here:

Submit Article

DOI: 10.32474 /GJAPM.2020.02.000147

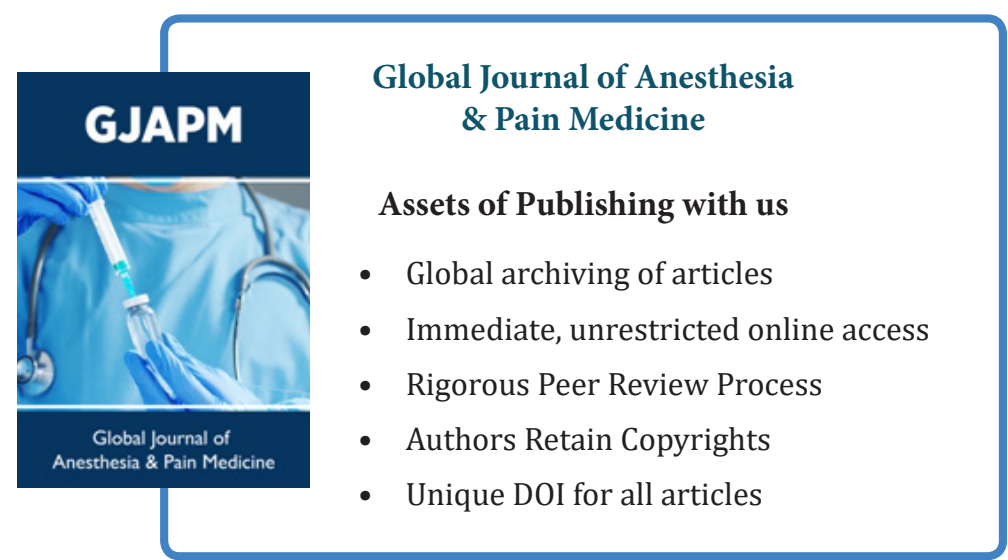

